\title{
OCCURRENCE OF FUNGI AND CYTOTOXICITY OF THE SPECIES: ASPERGILLUS OCHRACEUS, ASPERGILLUS NIGER AND ASPERGILLUS FLAVUS ISOLATED FROM THE AIR OF HOSPITAL WARDS
}

AGNIESZKA GNIADEK ${ }^{1}$, PAWEŁ KRZYŚCIAK², MAGDALENA TWARUŻEK ${ }^{3}$, and ANNA B. MACURA ${ }^{2}$

${ }^{1}$ Jagiellonian University Medical College, Kraków, Poland

Faculty of Health Sciences, Institute of Nursing and Midwifery, Department of Nursing Management and Epidemiology Nursing

${ }^{2}$ Jagiellonian University Medical College, Kraków, Poland

Faculty of Health Sciences, Chair of Microbiology, Department of Mycology

${ }^{3}$ Kazimierz Wielki University, Bydgoszcz, Poland

Faculty of Natural Sciences, Institute of Experimental Biology, Department of Physiology and Toxicology

\begin{abstract}
Objectives: The basic care requirement for patients with weakened immune systems is to create the environment where the risk of mycosis is reduced to a minimum. Material and Methods: Between 2007 and 2013 air samples were collected from various wards of a number of hospitals in Kraków, Poland, by means of the collision method using MAS-100 Iso MH Microbial Air Sampler (Merck Millipore, Germany). The air mycobiota contained several species of fungi, and almost 1/3 of it was made up of the species of the Aspergillus genus. Sixty-one strains of species other than A. fumigatus were selected for the research purposes, namely: 28 strains of $A$. ochraceus, 22 strains of $A$. niger and 11 strains of $A$. flavus species. Selected fungi underwent a cytotoxicity evaluation with the application of the MTT colorimetric assay (3-(4,5-dimethylthiazol-2-yl)-2,5diphenyltetrazolium bromide). The assay assesses cell viability by means of reducing the yellow tetrazolium salt to insoluble formazan. A semi-quantitative scale for cytotoxicity grading was adopted: low cytotoxic effect $(+)$ with half maximal inhibitory concentration $\left(\mathrm{IC}_{50}\right)$ for values ranging from $31.251 \mathrm{~cm}^{2} / \mathrm{ml}$ to $7.813 \mathrm{~cm}^{2} / \mathrm{ml}$, medium cytotoxic effect $(++)$ for values ranging from $3.906 \mathrm{~cm}^{2} / \mathrm{ml}$ to $0.977 \mathrm{~cm}^{2} / \mathrm{ml}$ and the high one $(+++)$ for values ranging from $0.488 \mathrm{~cm}^{2} / \mathrm{ml}$ to $0.061 \mathrm{~cm}^{2} / \mathrm{ml}$. The absence of cytotoxicity was determined when the $\mathrm{IC}_{50}$ values was at $\geq 50$. Results: For 48 samples the analyzed fungi displayed the cytotoxic effect with $A$. ochraceus in 26 out of 28 cases, with 11 strains displaying the high cytotoxic effect. The lowest cytotoxicity was displayed by fungi of $A$. niger in 13 out of 22 cases, and the major fungi of $A$. flavus species were toxic ( 9 out of 11 cases). Conclusions: A half of the fungi displayed the low cytotoxic effect. On the basis of the comparison of average cytotoxicity levels it was determined that there were significant differences in the levels of cytotoxicity of the analyzed fungi. However, such statement may not provide grounds for a definite conclusion about the compared species of fungi that display a more cytotoxic effect than others. Int J Occup Med Environ Health 2017;30(2):231-239
\end{abstract}

Key words:

Cytotoxicity, Airborne fungi, MTT assay, Aspergillus, Air sampling, Hospital environment

Excerpts were partially published during the Symposium "Power of fungi and mycotoxins in health and disease" in Primošten, Croatia, October 19-22, 2011. Received: November 4, 2015. Accepted: April 12, 2016.

Corresponding author: A. Gniadek, Jagiellonian University Medical College, Faculty of Health Sciences, Institute of Nursing and Midwifery, Department of Nursing Management and Epidemiology Nursing, Kopernika 25, 31-005 Kraków, Poland (e-mail: agnieszka.gniadek@uj.edu.pl). 


\section{INTRODUCTION}

Moulds with highest toxicity come from the Aspergillus genus and are considered to be most pathogenic for humans. Although species of Aspergillus non fumigatus, such as: A. flavus, $A$. ochraceus, $A$. niger, $A$. versicolor or $A$. terreus, are rarely aetiology factors of infections, they may be highly toxic [1-4].

It might be proven by other written sources that fungi belonging to Aspergillus fumigatus species are characterized by the highest toxicity among all the species belonging to this genus [5,6]. Aflatoxin B1, ochratoxin A as well as gliotoxin produced by the fungi belonging to $A$. fumigatus species are found to be responsible for such high toxicity. Fewer scientific publications are devoted to toxic fungi belonging to other species such as: A. flavus or A. ochraceus [7,8]. This trend may be explained by the fact that fungi belonging to A. fumigatus species are most frequently detected in the clinical material and, moreover, they are the ones which are usually responsible for fungal infections among immunocompromised patients [9-11]. Nevertheless, there are the research results that prove fungi which have not been considered pathogenic so far, e.g., A. terreus, to be the cause of opportunistic infections [12], too.

Available literature covering the subject does not clearly define to what extent the exposition to toxigenic airborne fungi may contribute to actual adverse effects on human health. However, it is generally agreed that the mould incidence in the buildings is unhealthy and in residential premises measures must be taken to limit mould development. Some researchers suggest that long-term exposition to toxigenic moulds may lead to disrupted natural killer (NK) cell activity displaying a whole range of neurological symptoms such as: headaches, general debilitating, fever, cough, memory loss, depression, mood swings, sleep disturbances, anxiety, chronic fatigue, and seizures [5].

Pieckova [6] underlines the importance of research regarding the mechanisms of the adverse effects of airborne moulds in the buildings over human health. Pieckova's [6] and Maličev et al.'s [13] works suggest the existence of the immunosuppressive influence and (bioaerosol-induced) inflammatory reaction to $\beta$-glucans from the cell walls of the moulds, toxic and irritating effect of their exoand endometabolites - mycotoxins, volatile organic compounds (VOCs) and fungal hemolysins. The aim of this research has been to define the occurrence and cytotoxicity of the fungi other than A. fumigatus (A. ochraceus, A. niger and $A$. flavus) in the hospital environment where patients with compromised immunity systems are hospitalized.

\section{MATERIAL AND METHODS}

Seven wards of a number of hospitals in Kraków, Poland, were selected for the assessment of the environment of the rooms where immunocompromised patients were hospitalized. The research was conducted in patients' room as well as in other functional areas of the hospital qualified as sanitary zones of general medical cleanliness and variable cleanliness zones, e.g., procedure rooms, bathrooms or hallways. The research was conducted in the following wards: Adult Intensive Care Unit (AICU), Neonatal Intensive Care Unit (NICU), Neonatal High Dependency Unit (NHDU), General and Systemic Oncology Ward (GSOW), Gynecological Oncology (GO), Hematology (post bone marrow transplants - H), Otolaryngology (patients diagnosed with laryngeal carcinoma-O).

The research input from each of the inspected rooms constituted air samples collected by means of the collision method. Samples of air were collected in each of the inspected wards during the period of 5 days with a bidaily sampling scheme (morning and evening). The whole research input was obtained in the period from November 2007 to April 2013 (sampling was performed mostly in the last quarter of a given calendar year). The only exception was the otolaryngology unit where samples were collected only in the periods when patients undergoing the procedure of surgical insertion of a tracheostomy tube due to the laryngeal carcinoma diagnosis were present. 
The samples were collected by means of the MAS-100 Iso MH Microbial Air Sampler (Merck Millipore, Germany). All of the measurements were performed in the same manner, i.e., during the collection times the air sampler was placed in the middle of the room at approximately $1.5 \mathrm{~m}$ above the floor level. Two hundred liters of air was subsequently collected directly onto the Sabouraud agar plate (bioMérieux, France). The total of 825 air samples were collected from all of the examined rooms. All the collected samples were incubated and, subsequently, the occurrence of fungi was assessed in the quantitative and qualitative aspects.

The first step of the cytotoxicity assessment procedure involved initial incubation of the fungi at $28^{\circ} \mathrm{C}$ for $96 \mathrm{~h}$. Grown fungal colonies were exposed to thermal shock by being placed in the temperature of $4^{\circ} \mathrm{C}$ for the subsequent 96 h. From the strains of moulds grown in the Petri dishes (Czapek-Dox medium) extracts were prepared to be later evaporated to dryness under a stream of nitrogen. Next, a series of test dilutions was prepared which were subsequently transferred onto swine kidney (SK) cells. The cytotoxicity studies were conducted in the Department of Physiology and Toxicology in the Institute of Experimental Biology of the Kazimierz Wielki University, Bydgoszcz, Poland.

Cytotoxicity testing was conducted by means of incubation of the extracts of the tested fungi with a cultivation of the pig renal cells for $48 \mathrm{~h}$. The cells were grown until $100 \%$ confluence was achieved in the medium containing a solution of antibiotics (Penicillin, Streptomycin (Sigma-Aldrich, USA)) and fetal bovine serum (SigmaAldrich, USA) in the $\mathrm{CO}_{2}$ Hera Cell Heraeus incubator $150 \mathrm{i}\left(5 \% \mathrm{CO}_{2}, 37^{\circ} \mathrm{C}\right.$, humidity at $\left.98 \%\right)$ (Thermo Fisher Scientific, USA). Cell culture suspension was prepared for the test in the volume of $2.2 \times 10^{5} \mathrm{ml}$ in the medium. The level of cytotoxicity was defined by means of the spectrophotometric analysis performed with the ELISA microplate reader (Digiscan by Asys Hitech $\mathrm{GmbH}$, Austria) and the MikroWin software (Mikrotek Laborsysteme GmbH, Germany) using a color change in the MTT tetrazolium salt (3-(4,5-dimethylthiazol-2-yl)-2,5-diphenyltetrazolium bromide) to formazan. The evaluation was performed at the wavelength of $510 \mathrm{~nm}$ [14].

Threshold toxicity level is the smallest concentration of the extract from a fungal colony that causes a drop in the sample absorption to values $<50 \%$ of the cell division activity, evaluated by means of the dilution method. Semi-quantitative scale for cytotoxicity grading was adopted: the low cytotoxic effect $(+)$ with a half of maximal inhibitory concentration $\left(\mathrm{IC}_{50}\right)$ values ranging from $31.251 \mathrm{~cm}^{2} / \mathrm{ml}$ to $7.813 \mathrm{~cm}^{2} / \mathrm{ml}$, the medium cytotoxic effect $(++)$ with values ranging from $3.906 \mathrm{~cm}^{2} / \mathrm{ml}$ to $0.977 \mathrm{~cm}^{2} / \mathrm{ml}$ and the high one $(+++)$ with values ranging from $0.488 \mathrm{~cm}^{2} / \mathrm{ml}$ to $0.061 \mathrm{~cm}^{2} / \mathrm{ml}$. The cytotoxic effect was absent if the extract concentration at $31.251 \mathrm{~cm}^{2} / \mathrm{ml}$ failed to inhibit the growth of the swine kidney (SK) (and if the percentage of extinction with respect to the control group was $\geq 50 \%$ ) [14].

\section{Statistical analysis}

The ANOVA test was used for the purpose of statistical formulation in order to show differences in the measurements (levels) of cytotoxicity between the individual species of the fungi. In view of the fact that significant differences were revealed between the average toxicity values of the studied groups, the Pareto criterion method was applied [15]. According to the Pareto method, a separate criterion was formulated for each of the cytotoxicity class: where the assessment took place between 2 species of fungi compared with each other in order to identify the more toxic one out of the compared species. Unconditional criteria and conditional criteria with respect to the 4 classes of cytotoxicity were defined (the absence of cytotoxic effect, low cytotoxic effect, medium cytotoxic effect and high cytotoxic effect). The assessment criteria for the "absence of cytotoxic effect" class is an unconditional criterion based on 
the assumption that every species of fungi with a lower likelihood of occurrence of fungal samples from the "absence of cytotoxic effect" class is more toxic. The criterion for the "low cytotoxic effect" is a conditional criterion based on the assumption at issue: if the cytotoxicity lower than the low cytotoxic effect, medium or high cytotoxic effect occurs in the compared pair of fungal species, the more toxic species is the one with the lower likelihood of occurrence of the fungal samples from the "low cytotoxic effect" class. The criterion for the fungi from the "medium cytotoxic effect" class is a conditional criterion based on the assumption that if the higher cytotoxicity than the medium cytotoxic effect, low cytotoxic effect or the absence of cytotoxic effect occurs in the compared pair of fungal species, the more toxic species is the one with the higher likelihood of occurrence of the fungal samples from the "medium cytotoxic effect" class. In order to assess the high cytotoxic effect, an unconditional criterion was defined, based on the assumption that the more cytotoxic species is the species with the higher likelihood of occurrence of the fungal sample from the "high cytotoxic effect" class.

The relationships among the compared cases of likelihood were studied on the basis of the confidence intervals of $95 \%$ confidence interval (CI) determined by means of the Wald test [16], separately for each of the compared cases of likelihood $\mathrm{p}<0.05$ was adopted in tests as a statistically significant threshold value.

\section{RESULTS}

The research was carried out by means of the MTT tetrazolium salt analysis on the group of fungi belonging to genus Aspergillus collected in various quantities (ranging 5-13) from various hospital wards. The qualitative assessment revealed that members of the genus Aspergillus accounted for 22-38\% of all the detected fungi. The most dominant species of this genus was Aspergillus fumigatus. Among other identified species of fungi there were the following: A. niger, A. flavus and A. ochraceus. Having marked the species of the Aspergillus genus in order to assess their cytotoxicity effect with the MTT colorimetric assay 61 strains of the following species were randomly selected: A. ochraceus, A. flavus and A. niger. The exact number of the obtained strains of fungi including their places of isolation is presented in the Table 1 .

The detailed overview of the results with the cytotoxicity grading by means of the MTT assay of 61 strains of fungi from A. ochraceus (28 strains), A. niger (22 strains) and from the $A$. flavus species (11 strains) are shown in the Table 2. The cytotoxicity of the analyzed fungi was proven in 48 cases $(78.7 \%)$. The cytotoxicity was most commonly confirmed with the strains of $A$. ochraceus with $92.9 \%$ of the studied strains proving to be cytotoxic and among them $22(36 \%)$ displayed medium and high cytotoxicity effect. Nine out of 11 studied strains from the A. flavus species (81.8\%) proved cytotoxic with 4 strains (44.4\%) displaying medium and high cytotoxicity effect. The cytotoxicity of the fungi from the $A$. niger species was shown in 13 out of 22 studied strains $(59.1 \%)$ with 5 cases in this group displaying medium and high cytotoxicity effect (38.5\%). The highest percentage of cytotoxic strains was identified among the fungi from the $A$. ochraceus species.

On the basis of the comparison of average cytotoxicity levels for individual species of the fungi, it was determined that there were significant differences in the levels of the cytotoxicity of the analyzed fungi. However, the said statement may not provide grounds for a clear determination which of the compared species of fungi displays a more cytotoxic effect than others. According to the estimations, a small number of samples: $28 \mathrm{~A}$. ochraceus, $22 \mathrm{~A}$. niger and $11 A$. flavus, respectively, were sufficient to determine with high certainty (Chi ${ }^{2}$ test: $\left.\mathrm{p}=0.004\right)$ that the analyzed 3 species display a significant variability with respect to cytotoxicity. Moreover, it was proven that the average likelihood of incidence of individual classes of cytotoxicity with respect to each class of cytotoxicity separately, implies 
Table 1. Strains of the fungi in air samples collected from various wards of hospitals in Kraków, Poland, 2007 and 2013

\begin{tabular}{|c|c|c|c|c|c|c|c|c|}
\hline \multirow[t]{2}{*}{ Species } & \multicolumn{7}{|c|}{$\begin{array}{l}\text { Strains in ward } \\
{[\mathrm{n}]}\end{array}$} & \multirow{2}{*}{$\begin{array}{l}\text { Total } \\
{[\mathrm{n}(\%)]}\end{array}$} \\
\hline & AICU & NICU & NHDU & GSOW & GO & $\mathrm{H}$ & $\mathrm{O}$ & \\
\hline Aspergillus ochraceus & 2 & 5 & 6 & 5 & 0 & 6 & 4 & $28(45.9)$ \\
\hline Aspergillus niger & 3 & 6 & 3 & 3 & 3 & 1 & 3 & $22(36.1)$ \\
\hline Aspergillus flavus & 1 & 2 & 0 & 2 & 2 & 0 & 4 & $11(18.0)$ \\
\hline Total & 6 & 13 & 9 & 10 & 5 & 7 & 11 & $61(100.0)$ \\
\hline
\end{tabular}

AICU - Adult Intensive Care Unit; NICU - Neonatal Intensive Care Unit; NHDU - Neonatal High Dependency Unit; GSOW - General and Systemic Oncology Ward; GO - Gynecological Oncology; H - Hematology (post bone marrow transplants); O - Otolaryngology.

Table 2. Characteristics of the fungi in air samples collected in hospitals in Kraków, Poland, 2007 and 2013

\begin{tabular}{|c|c|c|c|c|c|c|c|}
\hline \multirow[b]{2}{*}{ Species } & \multicolumn{4}{|c|}{ Level of cytotoxicity } & \multicolumn{3}{|c|}{ Threshold level of toxicity } \\
\hline & none & + & ++ & +++ & $\mathrm{M} \pm \mathrm{SD}$ & $\mathrm{Me}$ & $\begin{array}{c}\text { skewness/ } \\
\text { kurtosis }\end{array}$ \\
\hline $\begin{array}{l}\text { Aspergillus flavus } \\
\qquad(\mathrm{N}=11)\end{array}$ & & & & & $16.535 \pm 12.910$ & 15.63 & $00.70 /-1.83$ \\
\hline $\mathrm{n}(\%)$ & $2(18.20)$ & $5(45.40)$ & $3(27.30)$ & $1(9.10)$ & & & \\
\hline $95 \% \mathrm{CI}$ & $0.01-0.24$ & $0.21-0.72$ & $0.09-0.57$ & $0.00-0.40$ & & & \\
\hline $\mathrm{p}$ & 0.07 & 0.45 & 0.27 & 0.09 & & & \\
\hline $\begin{array}{l}\text { Aspergillus niger } \\
\qquad(\mathrm{N}=22)\end{array}$ & & & & & $19.41 \pm 13.02$ & 23.44 & $-0.39 /-1.65$ \\
\hline $\mathrm{n}(\%)$ & $9(40.90)$ & $8(36.40)$ & $2(9.10)$ & $3(13.60)$ & & & \\
\hline $95 \% \mathrm{CI}$ & $0.23-0.61$ & $0.20-0.57$ & $0.02-0.29$ & $0.04-0.34$ & & & \\
\hline $\mathrm{p}$ & 0.41 & 0.36 & 0.09 & 0.14 & & & \\
\hline $\begin{array}{l}\text { Aspergillus ochraceus } \\
\qquad(\mathrm{N}=28)\end{array}$ & & & & & $6.701 \pm 11.78$ & 0.98 & $1.70 / 1.07$ \\
\hline $\mathrm{n}(\%)$ & $2(7.1)$ & $4(14.3)$ & $11(39.3)$ & $11(39.3)$ & & & \\
\hline $95 \% \mathrm{CI}$ & $0.04-0.49$ & $0.05-0.32$ & $0.24-0.58$ & $0.24-0.58$ & & & \\
\hline $\mathrm{p}$ & 0.18 & 0.14 & 0.39 & 0.39 & & & \\
\hline
\end{tabular}

"+" - low cytotoxic effect; "++" - medium cytotoxic effect; "+++" - high cytotoxic effect.

$\mathrm{M}$ - mean; SD - standard deviation; Me - median; N - number of strains; CI - confidence interval.

the higher cytotoxicity levels of the fungi of the $A$. ochraceus species. These species of fungi display a higher cytotoxic effect when compared to $A$. flavus or the species of A. niger.

The statistical analysis of the confidence intervals showed that for each of the classes of the cytotoxicity separately, fungi of the A. ochraceus species either fail to display any significant differences from each of the 2 remaining species, or they are significantly more cytotoxic. Thus, according to the Pareto criterion, we may assume that fungi of the $A$. ochraceus species display the higher levels of cytotoxicity than the 2 remaining studied species of fungi. 


\section{DISCUSSION}

Toxicity studies conducted with fungal extracts allow for the analysis of noxious effects of metabolites unknown or excluded from several methodologies of mycotoxins research. The assessment includes both the components of the $\beta$-glucans cell wall and the substances found in the cytoplasm or various compounds released into the environment by the hyphae of the fungi [17-20]. On the basis of the obtained cytotoxicity assessment results of the extracts from the spores of 9 moulds, Schultz et al. draws the conclusions on the potential risk for health as the result of the toxic influence after a significant inhalation of a large number of mould spores [21]. Similar observations may be found in the works of Anyanwu et al. [5] and Pieckova [6], mentioned in the foreword for this article.

The species of fungi studied in this work are well known producers of mycotoxins: A flavus produces, inter alia, kojic acid, 3-nitropropionic acid, cyclopiazonic acid as well as aflatoxins: B1, B2, G1, G2. A. niger produces malformin, ochratoxin A or toxic oxalates. A. ochraceus may produce penicillin acid, A and B ochratoxins, xanthomegnin, viomellein and vioxanthin [22]. Literature offers very few studies that discuss the problems of cytotoxicity displayed by moulds found in the environment inhabited by humans. The said studies for the most part deal with the issue of the assessment of the cytotoxicity of the fungi from the genera of Penicillium sp., Stachybotrys sp., Chaetomium sp. or Aspergillus sp., which are commonly found in humid apartments [23-25] or marked in relation to the employment environment at various work stations [26].

Macedo et al. [27] evaluated the purity of air in the High Complexity Adult Intensive Care Unit (AICU), Newborn Intensive Care Unit (NICU) as well as in the hallways, corridors and external areas. They stated that the average number of spores did not exceed the Brazilian norm according to the National Agency for Sanitary Vigilance $\left(750 \mathrm{cfu} / \mathrm{m}^{3}\right)$ [27] in any of the buildings. According to most studies conducted by different scientific centers norms for the presence of fungi in the hospital rooms were very often exceeded [28-31]. However, literature devoted to the subject, apart from the author's own research [32,33] does not provide additional in-depth information on the subject of the assessment of the cytotoxicity of moulds that come from the environment of hospital rooms.

Certain reports may be found in scientific literature dealing with moulds cytotoxicity analyzed in the context of the manner of acquisition of fungi for studies. In vivo studies evaluating the cytotoxicity of collected airborne moulds from humid apartments in Scotland revealed that almost 1/2 (47\%) of the evaluated fungi displayed the cytotoxic effect [23].

In vitro studies evaluating the cytotoxicity of moulds found on building materials and infected by said moulds revealed that the cytotoxicity of the fungi was related to the type and concentrations of mycotoxins produced by the fungi occurring on building materials. The higher cytotoxic effect was displayed by the building materials affected with moulds rather than pure samples that contained mycotoxins in lower concentrations [34]. Thus, it may be assumed that the exposure to toxins produced by moulds on affected building materials may be a significant factor predisposing for infections caused by these moulds in sensitive patients. However, the research conducted by Fairs et al. [35] in order to evaluate the concentration of airborne $A$. fumigatus fungi in the rooms inhabited by asthma patients revealed that home environment should be treated as a potential source of exposure to moulds and may only predispose patients suffering from asthma to fungal colonization of airways, which, however, does not have to mean that the patient will get infected. Those studies find substantiation in the author's own research conducted in hospital environment on 13 subjects diagnosed with laryngeal and pharyngeal carcinoma who underwent the insertion of the tracheostomy tube. Tracheostomy tube colonization with airborne moulds occurring in the room was observed only in one case and involved fungi 
of the A. flavus species. Further research proved, though, that the said string was cytotoxic and displayed a low cytotoxic effect: $\mathrm{IC}_{50}=31.25 \mathrm{~cm}^{2} / \mathrm{ml}[36]$.

In their research Schulz et al. [21] showed that $\mathrm{IC}_{50}$ (as assessed by the MTT assay) for the spore extracts of $A$. niger was lower than for $A$. fumigatus [21]. Moreover, A. ochraceus string, also included in the said studies, displayed a low cytotoxicity effect, which was not proven in this research. Quite the opposite, the fungi of this species showed the higher cytotoxicity than the fungi of the $A$. $n$ ger or A. flavus species.

The author's other own research [33] and the results obtained by Kamei et al. [37] and Slesiona et al. [38] proved that $A$. fumigatus species displayed the higher cytotoxic effect than $A$. flavus, $A$. niger and $A$. terreus species did. No research was encountered, however, which would show that moulds of the $A$. ochraceus species displayed a higher cytotoxic effect than any other Aspergillus apart from $A$. fumigatus species did. However, this research showed that the 3 studied species: $A$. niger, $A$. flavus and $A$. ochraceus displayed a significant difference $(\mathrm{p}=0.004)$ with respect to their cytotoxicity. Moreover, it was proven that the average likelihood of incidence of individual classes of cytotoxicity: "absence of cytotoxic effect, low cytotoxic effect, medium cytotoxic effect, high cytotoxic effect" with respect to each class of the cytotoxicity separately, implies the higher cytotoxicity levels of the fungi of the $A$. ochraceus species when compared to the fungi of the $A$. flavus and $A$. niger species. Summing up, cytotoxic strains of moulds from $A$. ochraceus, $A$. niger or $A$. flavus present in the environment of immunocompromised patients may pose an additional risk for those patients.

\section{CONCLUSIONS}

Aspergillus genus and Aspergillus fumigatus were found to be the most prevailing species at hospital wards.

The comparison of the average cytotoxicity levels has proven that there are significant differences as far as the cytotoxicity levels of the fungi belonging to the $A$. ochraceus, $A$. flavus and $A$. niger species are concerned but it cannot serve the grounds for the conclusion which of the compared fungal species is more cytotoxic than the others.

More studies on this topic are needed to explain the importance of fungal cytotoxicity in the pathogens in nosocomial aspergillosis.

\section{REFERENCES}

1. Wierzbicka M, Podsiadło B, Janczarski M. [Invasive pulmonary aspergillosis caused by Aspergillus ochraceus]. Pneumonol Alergol Pol. 1997;65(3-4):254-60. Polish.

2. Fianchi L, Picardi M, Cudillo L, Corvatta L, Mele L, Trapè G, et al. Aspergillus niger infection in patients with haematological diseases: A report of eight cases. Mycoses. 2004;47 (3-4):163-7, https://doi.org/10.1111/j.1439-0507.2004.00960.x. 3. Orzechowski Xavier M, Comarú Pasqualotto A, da Penha Uchoa Sales M, Bittencourt Severo C, Peixoto Camargo JJ, Carlos Severo L. Invasive pulmonary aspergillosis due to a mixed infection caused by Aspergillus flavus and Aspergillus fumigatus. Rev Iberoam Micol. 2008;25(3):176-8, https://doi.org/ 10.1016/S1130-1406(08)70041-X.

4. Krishnan S, Manavathu EK, Chandrasekar PH. Aspergillus flavus: An emerging non-fumigatus Aspergillus species of significance. Mycoses. 2009;52(3):206-22, https://doi.org/ 10.1111/j.1439-0507.2008.01642.x.

5. Anyanwu EC, Campbell AW, Jones J, Ehiri J. The neurological significance of abnormal natural killer cell activity in chronic toxigenic mold exposures. ScientificWorldJournal. 2003;13(3):1128-37, https://doi.org/10.1100/tsw.2003.98.

6. Pieckova E. Adverse health effects of indoor moulds. Arh Hig Rada Toksikol. 2012;63(4):545-9, https://doi.org/ 10.2478/10004-1254-63-2012-2221.

7. Baranyi N, Despot DJ, Palágyi A, Kiss N, Kocsubé S, Szekeres A, et al. Identification of Aspergillus species in Central Europe able to produce G-type aflatoxins. Acta Biol Hung. 2015;66(3):339-47, https://doi.org/10.1556/018.66.2015.3.9. 
8. Cary JW, Ehrlich KC, Beltz SB, Harris-Coward P, Klich MA. Characterization of the Aspergillus ochraceoroseus aflatoxin/sterigmatocystin biosynthetic gene cluster. Mycologia. 2009;101(3):352-62, https://doi.org/10.3852/08-173.

9. Bizet J, Cooper CJ, Zuckerman MJ, Torabi A, MendozaLadd A. A bleeding colonic ulcer from invasive Aspergillus infection in an immunocompromised patient: A case report. J Med Case Rep. 2014;5(8):407, https://doi.org/10.1186/17521947-8-407.

10. Koutsounas I, Pyleris E. Isolated enteric aspergillosis in a non severely immunocompromised patient. Case report and literature review. Arab J Gastroenterol. 2015;16(2): 72-5, https://doi.org/10.1016/j.ajg.2015.06.008.

11. Küpeli E, Ulubay G, Bayram Akkurt S, Öner Eyüboğlu F, Sezgin A. Invasive pulmonary aspergillosis in heart transplant recipients. Exp Clin Transplant. 2015;13 Suppl 1:352-5, https://doi.org/10.6002/ect.mesot2014.P204.

12. Erdem E, Kandemir H, Arıkan-Akdağlı S, Esen E, Açıkalın A, Yağmur M, et al. Aspergillus terreus infection in a sutureless self-sealing incision made during cataract surgery. Mycopathologia. 2015;179(1-2):129-34, https://doi. org/10.1007/s11046-014-9829-2.

13. Maličev E, Chowdhury H, Maček P, Sepčić K. Effect of ostreolysin, an Asp-hemolysin isoform, on human chondrocytes and osteoblasts, and possible role of Asp-hemolysin in pathogenesis. Med Mycol. 2007;45(2):123-30, https://doi.org/ 10.1080/13693780601039615.

14. Hanelt M, Gareis M, Kollarczik B. Cytotoxicity of mycotoxins evaluated by the MTT-cell culture assay. Mycopathologia. 1994;128(3):167-74, https://doi.org/10.1007/BF01138479.

15. Fernández FR, Nickel S, Puerto J, Rodríguez-Chía MA. Robustness in the Pareto-solutions for the multi-criteria minisum location problem. J Multi-Crit Decis Anal. 2001;10(4):191-203, https://doi.org/10.1002/mcda.303.

16. Gareis M. Cytotoxicity testing of samples originating from problem buildings. In: Johanning E, editor. Fungi and bacteria in indoor air environments: Health effects, detection and remediation. Proceedings of the International Conference;
1994 Oct 6-7; Saratoga Springs, USA. Saratoga Springs: Eastern New York Occupational Health Program: 1994. p. 139-44.

17. Agresti A, Coull BA. Approximate is better than "Exact" for interval estimation of binomial proportions. Am Stat. 1998;52(2):119-26.

18. Gutarowska B, Piotrowska M. Methods of mycological analysis in buildings. Build Environ. 2007;42(4):1843-50, https:// doi.org/10.1016/j.buildenv.2006.02.015.

19. Liang Y, Zhao W, Xu J, Miler D. Characterization of 2 related exoantigens from the biodeteriogenic fungus Aspergillus versicolor. Int Biodeterior Biodegradation. 2011;65(1): 217-26, https://doi.org/10.1016/j.ibiod.2010.11.005.

20. Ostrosky-Zeichner L, Alexander BD, Kett DH, Vazquez J, Pappas PG, Saeki F, et al. Multicenter clinical evaluation of the $(1 \rightarrow 3) \beta$-D-glucan assay as an aid to diagnosis of fungal infections in humans. Clin Infect Dis. 2005;41(5):654-9, https://doi.org/10.1086/432470.

21. Schulz T, Senkpiel K, Ohgke H. Comparison of toxicity of reference mycotoxins and spore extracts of common indoor moulds. Int J Hyg Environ Health. 2004;207(3):267-77, https://doi.org/10.1078/1438-4639-00282.

22. Bräse S, Encinas A, Keck J, Nising C. Chemistry and biology of mycotoxins and related fungal metabolites. Chem Rev. 2009;109(9):3903-90, https://doi.org/10.1021/cr050001f.

23. Smith JE, Anderson JG, Lewis CW, Murad YM. Cytotoxic fungal spores in the indoor atmosphere of the damp domestic environment. FEMS Microbiol Lett. 1992;100(1-3): 337-43, https://doi.org/10.1111/j.1574-6968.1992.tb14061.x.

24. Twarużek M. [Biological assay (MTT, PremiRTest) in the evaluation of contamination of domestic environment by mold mycotoxins] [doctoral dissertation]. Olsztyn: University of Warmia and Mazury in Olsztyn; 2005. Polish.

25. Huttunen K, Rintala H, Hirvonen MR, Vepsäläinen A, Hyvärinen A, Meklin T, et al. Indoor air particles and bioaerosols before and after renovation of moisture-damaged buildings: The effect on biological activity and microbial flora. Environ Res. 2008;107(3):291-8, https://doi.org/10.1016/j.envres. 2008.02.008. 
26. Gutarowska B, Skóra J, Stępień Ł, Twarużek M, BłajetKosicka A, Otlewska A, et al. Estimation of fungal contamination and mycotoxin production at workplaces in composting plants, tanneries, archives and libraries. World Mycotoxin J. 2014;7(3):345-55, https://doi.org/10.3920/WMJ2013.1640.

27. Macedo JI, Kubota TH, Matsumoto LS, Giordani AT, Takayanagui AMM, Mendes AA, et al. Air quality in a hospital environment. Safety Secur Eng. 2013;134:737-47, https://doi.org/ 10.2495/SAFE130661.

28. Kim KY, Kim YS, Kim D. Distribution characteristics of airborne bacteria and fungi in the general hospital of Korea. Ind Health. 2010;48(2):236-43, https://doi.org/10.2486/ indhealth.48.236.

29. Reboux G, Bellanger AP, Roussel S, Grenouillet F, Sornin S, Piarroux R, et al. Indoor mold concentration in Eastern France. Indoor Air. 2009;19(6):446-53, https://doi.org/ 10.1111/j.1600-0668.2009.00598.x.

30. Falvey DG, Streifel AJ. Ten-year air sample analysis of $A s$ pergillus prevalence in a university hospital. J Hosp Infect. 2007;67(1):35-41, https://doi.org/10.1016/j.jhin.2007.06.008.

31. Krajewska-Kułak E, Łukaszuk C, Hatzopulu A, Bousmoukilia S, Terovitou Ch, Amanatidou A, et al. Indoor air studies of fungi contamination at the Department of Pulmonology and Internal Medicine in Kavala Hospital in Greece. Adv Med Sci. 2009;54(2):264-8, https://doi.org/10.2478/v10039-009-0048-X.

32. Gniadek A, Macura AB, Twarużek M, Grajewski J. Cytotoxicity of Aspergillus strains isolated from the neonatal intensive care unit environment. Adv Med Sci. 2010;54(2): 242-9, https://doi.org/10.2478/v10039-010-0039-y.

33. Gniadek A, Macura AB, Górkiewicz M. Cytotoxicity of $A s$ pergillus fungi isolated from hospital environment. Pol J Microbiol. 2011;60(1):59-63.

34. Gutarowska B. [Hyphate fungi in building materials. Increase and production of mycotoxins and allergens]. Sci Bull Technical University Lodz. 2010;1074:1-164. Polish.

35. Fairs A, Agbetile J, Bourne M, Hargadon B, Monteiro WR, Morley JP, et al. Isolation of Aspergillus fumigatus from sputum is associated with elevated airborne levels in homes of patients with asthma. Indoor Air. 2013;23(4):275-84, https:// doi.org/10.1111/ina.12020.

36. Gniadek A, Krzyściak P, Hawryszuk A, Macura AB, Brzostek T, Składzień J. Mycobiota of the air in hospital rooms and the fungal colonisation of tracheostomy tubes used by patients diagnosed with larynx cancer - Preliminary research. Ann Parasitol. 2013;59(2):69-71.

37. Kamei K, Watanabe A, Nishimura K, Miyaji M, Cytotoxicity of Aspergillus fumigatus culture filtrate against macrophages. Nihon Ishinkin Gakkai Zasshi. 2002;43(1):37-41, https://doi. org/10.3314/jjmm.43.37.

38. Slesiona S, Gressler M, Mihlan M, Zaehle C, Schaller M, Barz D, et al. Persistence versus escape: Aspergillus terreus and Aspergillus fumigatus employ different strategies during interactions with macrophages. PLoS One. 2012;7(2):e31223, https://doi.org/10.1371/journal.pone.0031223.

This work is available in Open Access model and licensed under a Creative Commons Attribution-NonCommercial 3.0 Poland License - http://creativecommons.org/ licenses/by-nc/3.0/pl/deed.en. 